\title{
Cardiogenic Shock, Acute Severe Mitral Regurgitation and Complete Heart Block After Cavo-Tricuspid Isthmus Atrial Flutter Ablation
}

\author{
Thein Tun Aung, ${ }^{a}$, , Edward Samuel Roberto ${ }^{a}$, b, d, Kevin D. Kravitz, b, c
}

\begin{abstract}
Radiofrequency (RF) ablation is the first-line management of cavotricuspid isthmus dependent atrial flutter. It has been performed with $95 \%$ success rate. Adverse events are very rare. We report the first case of acute severe mitral regurgitation (MR) and complete heart block developed after successful atrial flutter ablation. A 62-year-old female with mild MR presented with palpitations. Surface electrocardiogram was suggestive of isthmus dependent atrial flutter. A duodecapolar mapping catheter showed an atrial flutter with cycle length of $280 \mathrm{~ms}$. An $8 \mathrm{~mm}$ tipped Thermistor RF ablation catheter was placed at the cavo-tricuspid isthmus. RF energy was delivered as the catheter was dragged to the inferior vena cava. Temperature limit was 60 ${ }^{\circ} \mathrm{C}$; the power output limit was $60 \mathrm{~W}$. The patient converted to sinus rhythm with the first ablation line. Bi-directional block was recorded. Two additional ablation lines lasting $60-120 \mathrm{~s}$ were delivered. The patient started having chest pain and developed complete heart block with no escape rhythm. She became hypotensive and was immediately paced from the right ventricle. There were no signs of pericardial tamponade. Emergent bedside echo demonstrated severe MR with a retracted posteromedial mitral valve leaflet. She was 100\% paced and EKG changes could not be assessed. Based on the sudden onset chest pain, hypotension, complete heart block and acute severe MR after ablation, the right coronary artery occlusion was suspected. She was immediately transferred to the catheterization laboratory. Coronary angiography revealed a total occlusion of the posterolateral branch from the right coronary artery. Balloon angioplasty and coronary artery stenting was performed. Complete heart block subsequently resolved. Subsequent bedside echocardiogram showed marked improvement of the MR. Patients with smaller body size have smaller hearts and more likely to have injury from RF current. Higher energy penetrates deeper and causes more tissue damage. The use of lower temperature limits $\left(55^{\circ} \mathrm{C}\right)$ and lower energy $(60 \mathrm{~W})$ for small, elderly, and female patients is encouraged.
\end{abstract}

Manuscript accepted for publication March 14, 2017

a'Wright State University Boonshoft School of Medicine, Dayton, OH, USA ${ }^{\mathrm{b}}$ Good Samaritan Hospital, Dayton, OH, USA

${ }^{\mathrm{c}}$ Dayton Heart and Vascular Hospital, Dayton, OH, USA

${ }^{\mathrm{d} C}$ Corresponding Author: Edward Samuel Roberto, Wright State University Boonshoft School of Medicine, Dayton, OH, USA.

Email:samroberto@mac.com

doi: https://doi.org/10.14740/cr534w
Keywords: Atrial flutter; Ablation; Complications; Cardiogenic shock; Mitral regurgitation; Complete heart block

\section{Introduction}

Atrial flutter can present significant clinical challenge to control with medical management alone. Radiofrequency (RF) ablation has emerged as first-line therapy in the management of cavo-tricuspid isthmus dependent atrial flutter. Since the 1990 s, it has been widely performed with success rates of above $95 \%$. Adverse events and complications are very rare. We report here the first case of cardiogenic shock, acute severe mitral regurgitation (MR) and complete heart block due to occlusion of the posterolateral branch of the right coronary artery immediately following RF ablation of atrial flutter.

\section{Case Report}

A 62-year-old female with a history of recurrent symptomatic atrial flutter status post electrical cardioversion 4 years ago presented for RF ablation. Her CHADSVASc score was zero and she had not been anticoagulated. She had mild mitral regurgitation noted on previous echocardiograms. She had history of Hodgkin's lymphoma, and hypothyroidism status post thyroidectomy.

Surface electrocardiogram (EKG) suggested isthmus dependent typical atrial flutter with negative $P$ waves in inferior leads and positive in V1 (Fig. 1). Transesophageal echocardiogram prior to the ablation showed mild MR, a mildly enlarged left atrium and normal left ventricle systolic function. No thrombus was seen in the left atrial appendage and its emptying velocity was $40 \mathrm{~cm} / \mathrm{s}$.

A duodecapolar mapping catheter was positioned with the distal poles in the coronary sinus and the mid poles along the cavo-tricuspid isthmus. Atrial flutter with cycle length of 280 ms was recorded. An 8 Fr quadripolar deflectable $8 \mathrm{~mm}$ tipped thermistor electroanatomic mapping RF ablation catheter (Navistar) was advanced into the right ventricle and placed at the distal aspect of cavo-tricuspid isthmus. A three-dimensional map of right atrium, isthmus and inferior vena cava (IVC) region was made. The ablation catheter was advanced into the 


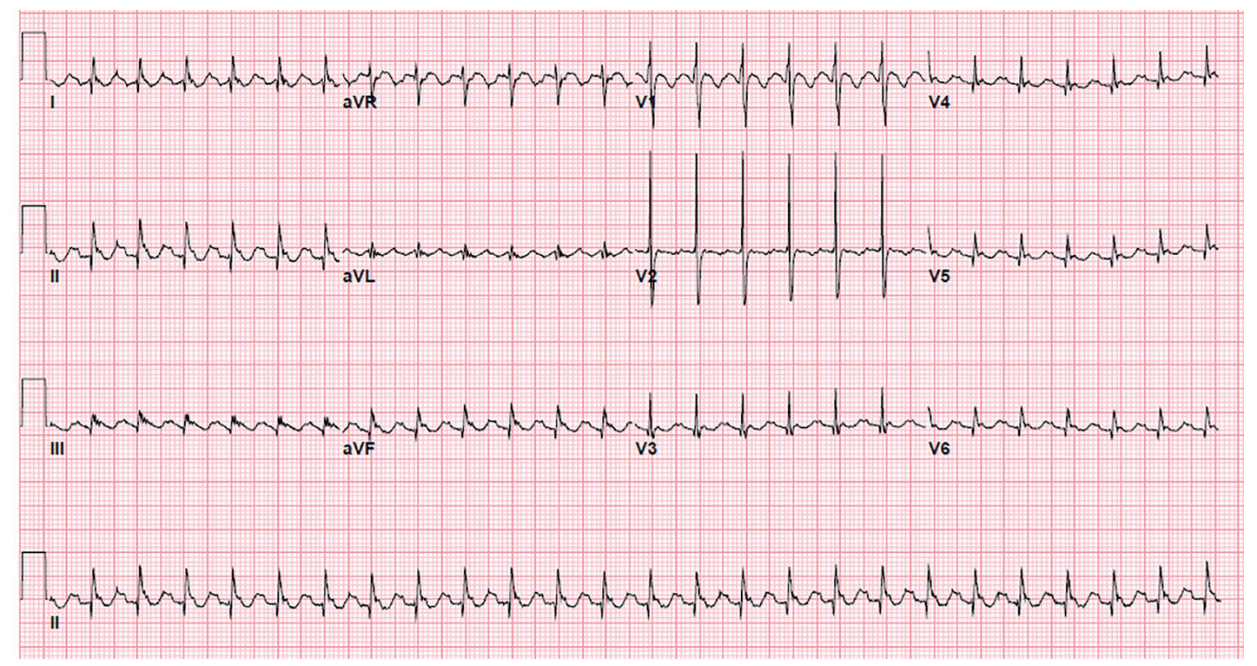

Figure 1. 12-lead surface electrogram showing saw tooth flutter waves: negative $P$ waves in inferior leads and positive $P$ waves in lead V1 and V2, suggestive of cavo-tricuspid isthmus dependent atrial flutter.

right ventricle and placed at the distal aspect of cavo-tricuspid isthmus.

$\mathrm{RF}$ energy was delivered as the catheter was dragged to the IVC. Maximum temperature limit was $60{ }^{\circ} \mathrm{C}$ and power output was limited to $60 \mathrm{~W}$. With the first ablation line lasting $120 \mathrm{~s}$, cessation of the atrial flutter was documented (Fig. 2 ). She converted to sinus rhythm. Bidirectional block was recorded. Two additional ablation lines lasting 60 - $120 \mathrm{~s}$ were delivered, for a total of three ablation lines. No additional RF lesions were planned.

At this point, the patient started complaining of chest dis- comfort and became hypotensive. Complete heart block was recorded on the monitor. She was immediately paced from the right ventricle. Despite this, her chest pain and hypotension worsened. Fluoroscopy showed no change in the cardiac silhouette. There were no clinical signs of pericardial tamponade. Stat bedside echo showed trivial pericardial fluid with normal left ventricular wall motion. However, there was wide open severe MR which had acutely developed (Fig. 3). Upon closer inspection of the mitral valve, the posteromedial leaflet was retracted and the regurgitant jet was posteriorly directed.

At this point the patient was $100 \%$ paced and EKG chang-

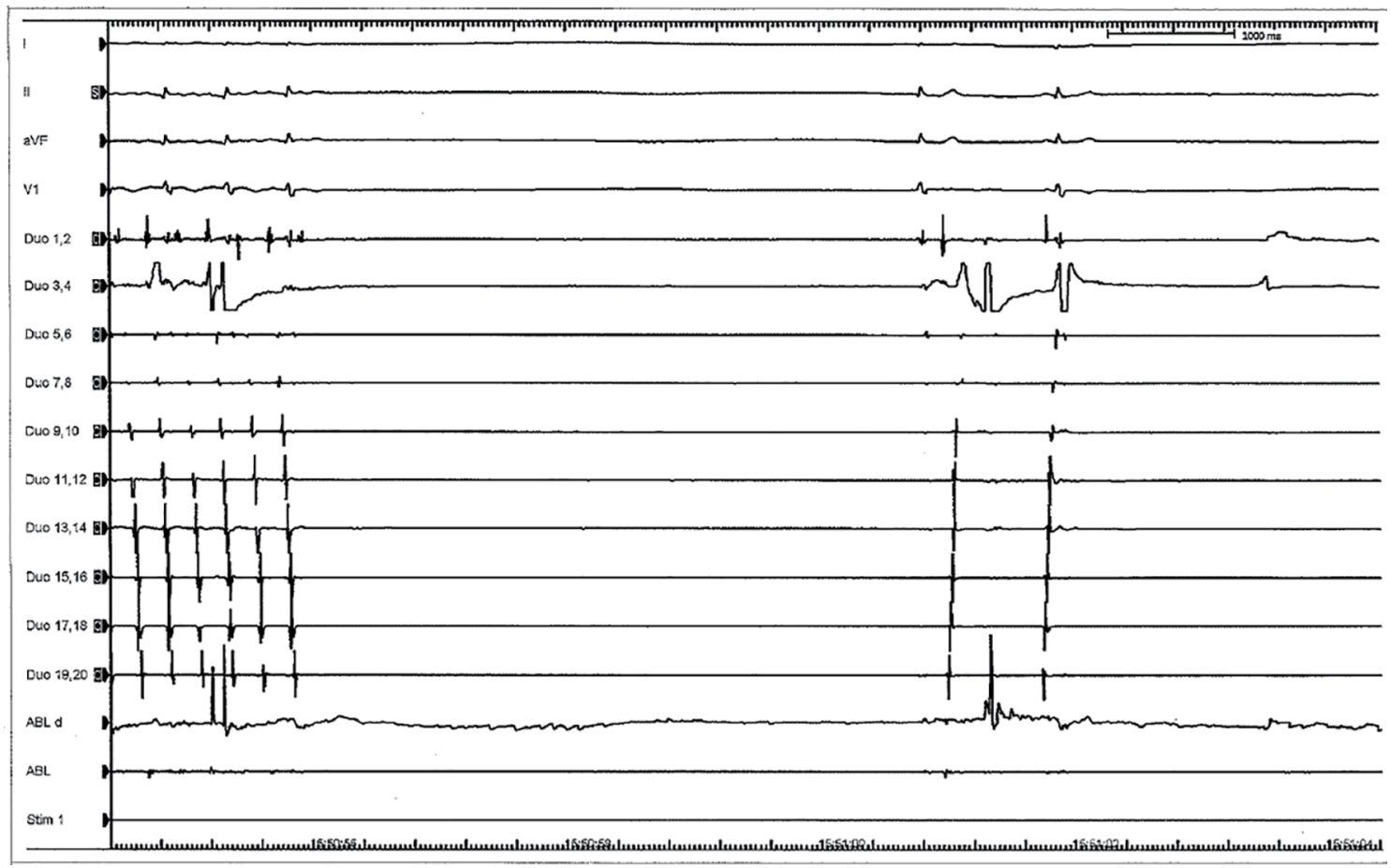

Figure 2. Cessation of atrial flutter after radiofrequency ablation and restoration of sinus rhythm. 


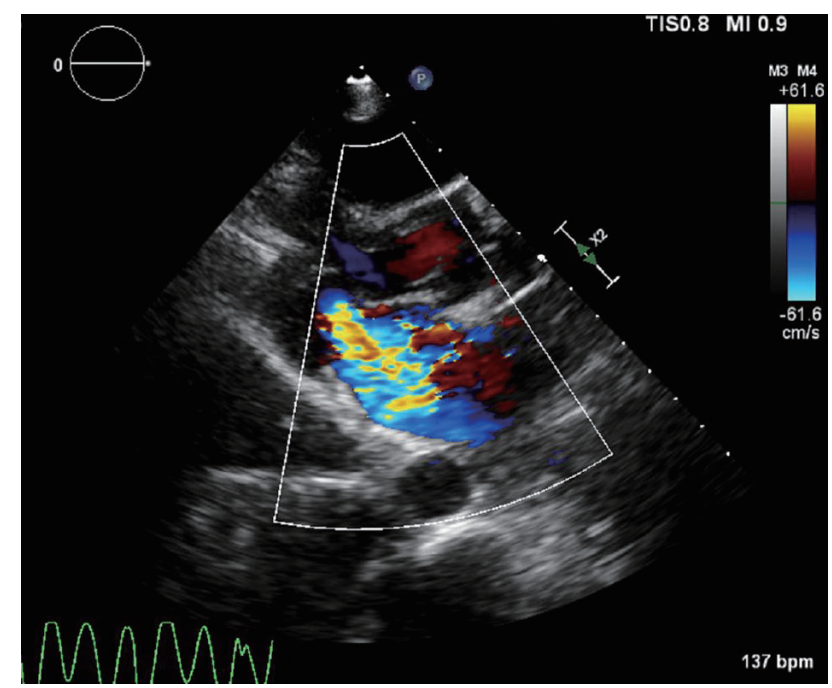

Figure 3. Acute severe (wide open) mitral regurgitation with retracted posteromedial leaflet.

es could not be assessed. However, based on the sudden development of chest pain, hypotension, complete heart block and acute severe MR, right coronary artery occlusion was suspected. The decision was made to evaluate her coronaries immediately. The ablation catheter was removed and a temporary transvenous pacemaker was inserted. The patient was also intubated due to decompensating respiratory status. She was transferred immediately to the cardiac catheterization laboratory.

Coronary angiography demonstrated patent left main, left anterior descending, and left circumflex arteries. However, the posterolateral branch from right coronary artery was totally occluded (Fig. 4). A 0.014 inch run-through wire was inserted into the culprit branch and angioplasty was done with $8 \times 15$

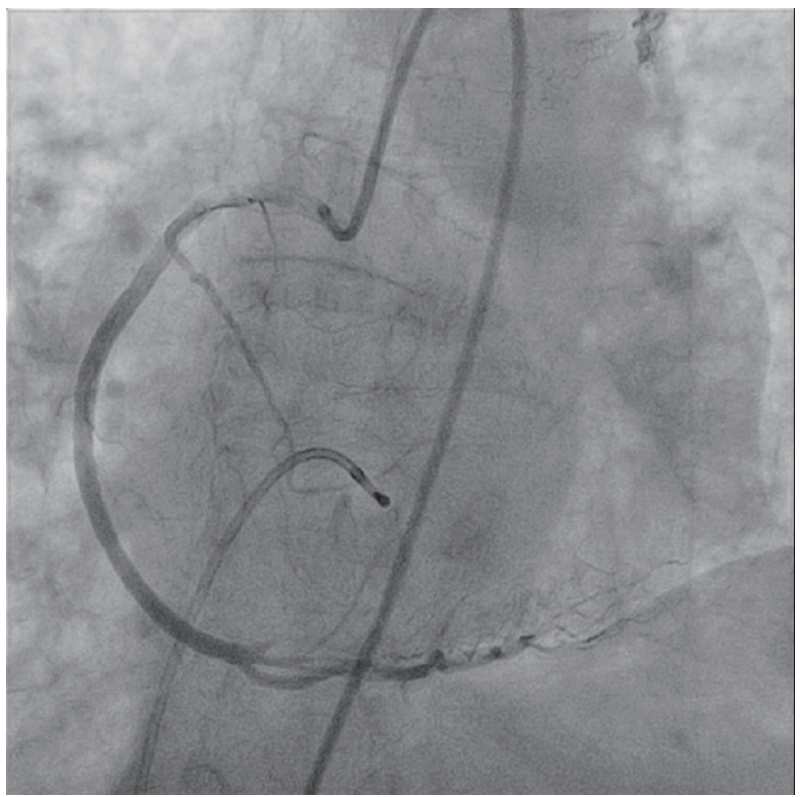

Figure 4. Complete occlusion of the posterolateral branch of right coronary artery.

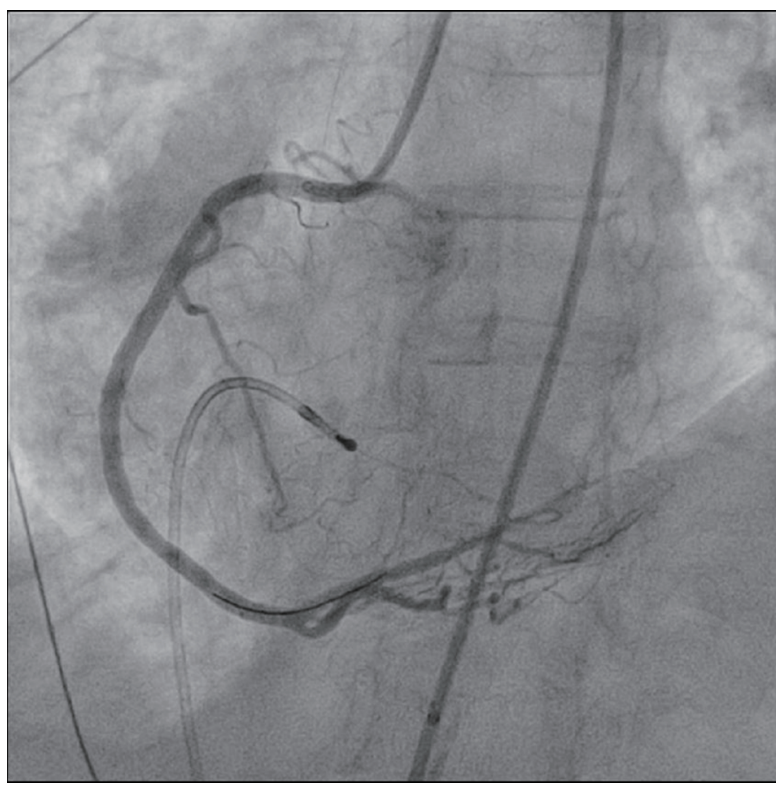

Figure 5. After coronary stenting, flow is re-established in occluded posterolateral branch.

mm Quantum Apex balloon. Complete heart block resolved and sinus tachycardia was noted after the blood flow was restored. With balloon angioplasty alone there was marked recoil of the stenosis and heart block recurred.

The decision was made to insert a drug eluting stent for further structural support. An everolimus-drug eluting platinum chromium coronary stent (Promus stent) was deployed resulting in restoration of blood flow to the posterolateral branch (Fig. 5). Persistent sinus rhythm was restored as well. Subsequent bedside echocardiogram showed marked improvement of MR. The patient's condition improved afterwards. Vasopressors were weaned. She was extubated the next day and discharged after 5 days.

\section{Discussion}

RF ablation of cavo-tricuspid isthmus dependent (typical) atrial flutter has been performed widely since 1990s. The conventional method (using $8 \mathrm{~mm}$ tip electrode catheter with power limit of $60-100 \mathrm{~W}$ and temperature limit of $50-60{ }^{\circ} \mathrm{C}$ ) has shown very high success rates, above $95 \%$, with very low adverse event rates.

Animal studies have shown up to $7 \mathrm{~mm}$ deep lesions are expected with $\mathrm{RF}$ ablation using $8 \mathrm{~mm}$ tip electrode catheters [1]. Right coronary artery occlusion has been demonstrated in studies with pigs. Bokenkamp et al postulated the factors associated with increased risk as follows: 1) heart size: smaller hearts have higher chance for adverse events; 2) long energy exposure, larger electrode; 3) anatomical variation, short distance between ablation site and right coronary artery [2].

One single center study from France retrospectively analyzed the records of 883 patients who underwent atrial flutter ablations done over 16 years. Only $1.7 \%$ of the patients, 14 out 


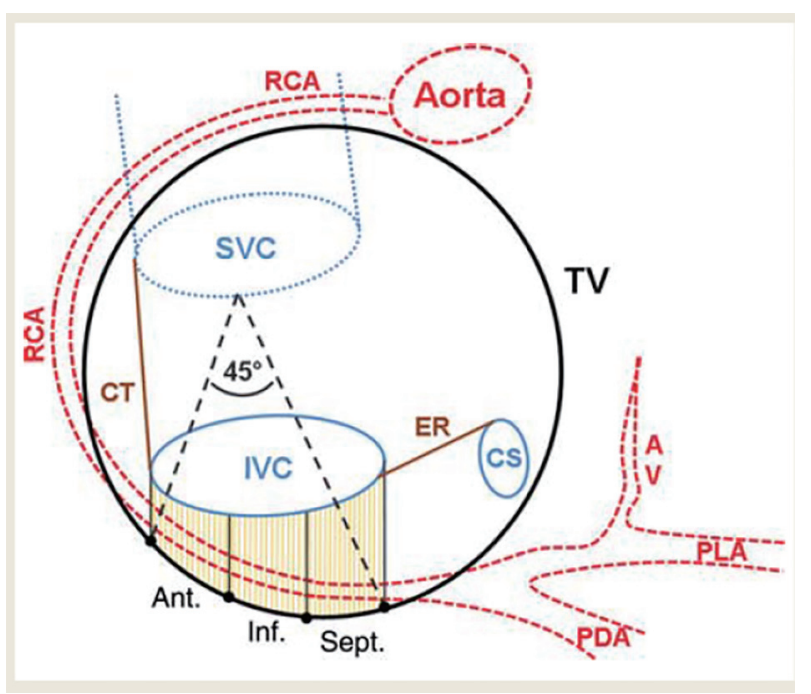

Figure 6. Schematic drawing of the CTI and course of the RCA. The center of the RCA was tagged in three dimensions. The CTI was defined by the insertion of the IVC to the right atrium and the tricuspid valve which were also tagged. The distance of the RCA to the CTI was calculated for the anterior (ant.), inferior (inf.), and septal (sept.) parts. In addition, the deviation of the RCA from the tricuspid valve plane towards the atrial aspect was calculated. SVC: superior vena cava; CT: crista terminalis; ER: Eustachian ridge; CS: coronary sinus; AV: atrioventricular nodal branch; PDA: posterior descending artery; PLA: posterolateral artery; IVC: inferior vena cava [7].

of 883 patients, had serious adverse events such as complete heart block, ventricular tachycardia, and coronary ischemia. There was only one patient who developed right coronary artery occlusion with complete heart block [3].

The pathophysiology of coronary occlusion during RF ablation is presumed to be thermal injury from RF energy [4]. Thrombosis from endothelial damage is possible as well. However, all the reported cases in literature, including our patient, demonstrated non-obstructive atherosclerosis in coronary angiograms. Regardless of the mechanism by which thermal injury causes coronary occlusion or thromboembolism, the management is urgent cardiac catheterization and percutaneous coronary intervention. Based on these facts, some authors stressed the importance of having interventional cardiologists as backup during the electrophysiology procedures [5].

Electro-anatomical mapping was done in our patient. Euro-flutter investigators mentioned mapping can shorten fluoroscopy time with the price of increased cost [6]. Caudal projections and bi-plane views help to recognize inadvertent septal or lateral RF application.

Figure 6 shows the anatomy of tricuspid isthmus and how the right coronary artery runs closely behind the tricuspid annulus [7]. In certain individuals, the thickness of right ventricular myocardium can be less than $5 \mathrm{~mm}$. The coronary sinus wall is even thinner than $5 \mathrm{~mm}$. Ouali et al reported right coronary artery occlusion during ablation. They noticed acute ST segment elevation on surface EKG from an inferior myocardial infarction which involved the middle segment of the right coronary artery [8].

In our patient, ST segment elevation could not be assessed.
She had complete heart block and required $100 \%$ pacing. After the coronary stent was deployed and sinus rhythm was restored, no inferior ST elevation was appreciated. Reasons that ST elevation was not seen include resolution of ischemia, the relatively small area of myocardium involved as well as the location of the ischemic myocardium. Still, the patient's hemodynamic status deteriorated very rapidly and suddenly resulting in cardiogenic shock. The patient's coronary anatomy explains this. The posterolateral branch of the right coronary artery was the single blood supply to the posterior mitral valve leaflet and supplied the AV nodal artery as well. Occlusion of the posterolateral right ventricular branch thus resulted in acute severe MR and complete heart block. Both of these were completely reversed after coronary intervention.

\section{Conclusion}

RF ablation of isthmus dependent atrial flutter is a commonly performed procedure. Although it is considered a relatively safe procedure, unforeseen complications can arise. Patients with smaller body size, such as women and the elderly, may have smaller hearts and thinner cardiac structures. Because of this, they are more likely to sustain unintended injury to adjacent cardiac structures when using RF energy. Higher power penetrates deeper and causes more tissue damage. Although there are no randomized clinical trials, the use of lower temperature limits $\left(55^{\circ} \mathrm{C}\right)$ and lower energy $(60 \mathrm{~W})$ for small, elderly patients should be encouraged. Still, unforeseen complications can arise due to anatomic variations of the right coronary artery and its branches. The operating electrophysiologist must always be cognizant of these variations and their associated risks, and be prepared to act timely to prevent further deterioration. Because there is risk for coronary complications, interventional cardiology should be available.

\section{Conflicts of Interest}

All authors declare no conflicts of interest.

\section{Funding}

No sources of external funding.

\section{References}

1. Otomo K, Yamanashi WS, Tondo C, Antz M, Bussey J, Pitha JV, Arruda M, et al. Why a large tip electrode makes a deeper radiofrequency lesion: effects of increase in electrode cooling and electrode-tissue interface area. J Cardiovasc Electrophysiol. 1998;9(1):47-54.

2. Paul T, Bokenkamp R, Mahnert B, Trappe HJ. Coronary artery involvement early and late after radiofrequency current application in young pigs. Am Heart J. 
1997;133(4):436-440.

3. Brembilla-Perrot B, Filali ML, Zinzius PY, Sellal JM, Beurrier D, Schwartz J, C DEC, et al. Is ablation of atrial flutter always safe? Pacing Clin Electrophysiol. 2012;35(9):1061-1066.

4. Mykytsey A, Kehoe R, Bharati S, Maheshwari P, Halleran S, Krishnan K, Razminia M, et al. Right coronary artery occlusion during RF ablation of typical atrial flutter. J Cardiovasc Electrophysiol. 2010;21(7):818-821.

5. Brembilla-Perrot B, Filali ML, Beurrier D, Groben L, Cedano J, Abdelaal A, Louis P, et al. Complete atrioventricular block during ablation of atrial flutter. Pacing Clin Electrophysiol. 2010;33(4):516-519.

6. Hindricks G, Willems S, Kautzner J, De Chillou C,
Wiedemann M, Schepel S, Piorkowski C, et al. Effect of electroanatomically guided versus conventional catheter ablation of typical atrial flutter on the fluoroscopy time and resource use: a prospective randomized multicenter study. J Cardiovasc Electrophysiol. 2009;20(7):734-740.

7. Klemm HU, Weber TF, Johnsen C, Begemann PG, Meinertz T, Ventura R. Anatomical variations of the right coronary artery may be a source of difficult block and conduction recurrence in catheter ablation of common-type atrial flutter. Europace. 2010;12(11):1608-1615.

8. Ouali S, Anselme F, Savoure A, Cribier A. Acute coronary occlusion during radiofrequency catheter ablation of typical atrial flutter. J Cardiovasc Electrophysiol. 2002;13(10):1047-1049. 